\title{
Criminologie
}

\section{Le contrôle plurinormatif des gangs de rue}

Contributions des études sur la délinquance des élites à la

compréhension de la judiciarisation de la criminalité de rue

\section{The plurinormative control of street gangs}

Contributions of elite deviance studies to the understanding of

the judicialization of street crimes

\section{El control plurinormativo de las pandillas callejeras \\ Contribuciones de los estudios sobre la delincuencia de las \\ elites a la comprehensión de la judiciarización de la \\ criminalidad callejera}

\section{João Velloso}

Volume 49, numéro 1, printemps 2016

Quand le crime économique contribue au développement des sciences sociales

URI : https://id.erudit.org/iderudit/1036198ar

DOI : https://doi.org/10.7202/1036198ar

Aller au sommaire du numéro

Éditeur(s)

Les Presses de l’Université de Montréal

ISSN

0316-0041 (imprimé)

1492-1367 (numérique)

Découvrir la revue

\section{Citer cet article}

Velloso, J. (2016). Le contrôle plurinormatif des gangs de rue : contributions des études sur la délinquance des élites à la compréhension de la judiciarisation de la criminalité de rue. Criminologie, 49(1), 153-178.

https://doi.org/10.7202/1036198ar

\section{Résumé de l'article}

Edwin Sutherland a été l'un des premiers à remettre en question l'association entre pauvreté et criminalité qui dominait le champ criminologique au début $\mathrm{du} \mathrm{xx}^{\mathrm{e}}$ siècle. Il proposait non seulement que la criminologie étudie les crimes en col blanc (white-collar crimes), mais également que ces études renforcent sa théorie de l'association différentielle en tant que théorie générale, applicable à tous les types de comportements criminels. Dans cet article, nous poursuivons un objectif similaire, mais à propos du contrôle social : nous proposons de réfléchir aux formes les plus visibles de la délinquance, et plus particulièrement à la criminalité de rue, à partir de certaines études sur la réaction sociale à la délinquance des élites. Nous suggérons que la notion d'« illégalismes privilégiés » (Acosta, 1988) est appropriée pour analyser tous les illégalismes. En effet, à l'instar des crimes en col blanc, les crimes de rue sont aussi fréquemment administrés par les institutions juridiques de façon plurinormative, c'est-à-dire en s'appuyant sur différents systèmes normatifs au-delà et en plus de la justice criminelle (par exemple, le droit administratif, le droit civil, etc.). Dans les faits, cette technique a souvent pour effet de punir davantage les délinquants visés, surtout les étrangers. 


\title{
Le contrôle plurinormatif des gangs de rue
}

\author{
Contributions des études sur la délinquance \\ des élites à la compréhension de la \\ judiciarisation de la criminalité de rue
}

\author{
João Velloso ${ }^{1}$ \\ Professeur adjoint \\ Faculté de droit, Section de common law, Université d'Ottawa \\ Joao.Velloso@uottawa.ca
}

RÉSUMÉ • Edwin Sutherland a été l'un des premiers à remettre en question l'association entre pauvreté et criminalité qui dominait le champ criminologique au début du $x x^{e}$ siècle. Il proposait non seulement que la criminologie étudie les crimes en col blanc (white-collar crimes), mais également que ces études renforcent sa théorie de l'association différentielle en tant que théorie générale, applicable à tous les types de comportements criminels. Dans cet article, nous poursuivons un objectif similaire, mais à propos du contrôle social: nous proposons de réfléchir aux formes les plus visibles de la délinquance, et plus particulièrement à la criminalité de rue, à partir de certaines études sur la réaction sociale à la délinquance des élites. Nous suggérons que la notion d'«illégalismes privilégiés» (Acosta, 1988) est appropriée pour analyser tous les illégalismes. En effet, à l'instar des crimes en col blanc, les crimes de rue sont aussi fréquemment administrés par les institutions juridiques de façon plurinormative, c'est-à-dire en s'appuyant sur différents systèmes normatifs au-delà et en plus de la justice criminelle (par exemple, le droit administratif, le droit civil, etc.). Dans les faits, cette technique a souvent pour effet de punir davantage les délinquants visés, surtout les étrangers.

MOTS CLÉS - Illégalismes privilégiés, délinquance des élites, membres de gangs de rue, contrôle d'immigration, réaction sociale.

1. Faculté de droit, Section de common law, Université d'Ottawa, 57, rue Louis-Pasteur (Pavillon Fauteux), Ottawa (Ontario), Canada, K1N 6N5.

Criminologie, vol. 49, nº 1 (2016) 


\section{Introduction}

Cet article ne porte pas sur la délinquance des élites à proprement parler, mais plutôt sur la criminalité de rue. En réponse à la question posée par ce numéro spécial, soit celle de la contribution du crime économique aux sciences sociales, et particulièrement à la criminologie, nous suggérons que les études sur la délinquance des élites nous permettent de poser un autre regard sur le contrôle d'autres formes de déviance, telles que le contrôle de l'immigration, le contrôle des manifestants et même celui des gangs de rue, des comportements qui, à première vue, ne sont pas perçus comme des crimes économiques. Nous chercherons à démontrer dans quelle mesure les études sur la criminalité économique, un domaine de recherche encore relativement marginal au sein de la criminologie, peuvent contribuer de façon originale à l'étude de certains thèmes dominants et centraux de notre discipline. Nous sommes d'avis que ces études offrent non seulement une contribution d'ordre empirique et thématique, mais également une contribution théorique à la compréhension des autres «crimes» et à la criminologie plus largement.

De façon plus spécifique, nous indiquons que les études criminologiques sur la réaction sociale à la délinquance des élites fournissent les outils nécessaires pour penser la criminologie d'une façon plurinormative, c'est-à-dire en se référant non seulement à la justice criminelle, mais également à d'autres systèmes normatifs tels que le droit administratif, le droit civil, le droit du travail, etc. Ce faisant, les travaux portant sur la réaction sociale à la délinquance des élites proposent une rupture avec une forme de savoir consolidée au Xix ${ }^{e}$ siècle (Debuyst, Digneffe et Pires, 1998), soit la criminologie, qui encore aujourd'hui, définit son objet d'étude quasi exclusivement en fonction des crimes et des peines (Velloso, 2013a). En laissant supposer que les études sur la délinquance des élites apportent un éclairage à la criminalité de rue, il devient possible de percevoir que les situations-problèmes (Hulsman, 1986; Hulsman et De Celis, 1982)² traditionnellement classifiées comme des crimes par la criminologie ne sont en fait pas tellement différentes des crimes en col blanc du point de vue de la réaction sociale. En d'autres mots, les crimes de rue ne constituent pas forcément ou exclusivement

2. Slingeneyer (2005) résume bien la notion de situation-problème en précisant qu'elle est une idée clé pour la pensée abolitionniste hulsmanienne et pour la critique de leur notion de crime (voir p. 24-27). 
des crimes, mais ils doivent plutôt ou également être perçus comme des illégalismes dans un sens plus large. Nous nous trouvons ainsi de plus en plus souvent devant des situations-problèmes qui sont susceptibles d'être judiciarisées de multiples façons, ce qui transforme ces situations à la fois en crimes et en non-crimes, et ce, parfois simultanément.

Nous développerons d'abord notre argument théorique. Dans la première section, intitulée La criminologie avant la criminalité en col blanc, nous montrons que Sutherland avait déjà associé d'une certaine façon, la délinquance des élites aux autres formes de comportements criminels dans ses écrits sur la criminalité en col blanc (Sutherland, 1940, 1941, 1945, 1983), alors que dans la seconde section, intitulée La renaissance de la criminalité en col blanc dans les années 1980, nous nous concentrons plutôt sur la réaction sociale à la délinquance des élites et sur la notion de gestion différentielle des illégalismes développée par Foucault (1975, 2013), Lascoumes (1983, 1985, 1986, 1996), Shapiro (1980, 1984, $1985)$ et Acosta $(1984,1985,1988)$. Nous nous tournons ensuite, en troisième partie, vers quelques exemples empiriques. Dans la section $D e$ près, les chats ne sont pas toujours gris, nous présentons quelques cas de gestion différentielle des situations-problèmes associées à la criminalité de rue. Ce faisant, nous souhaitons problématiser l'idée selon laquelle ces illégalismes sont «privilégiés» sur le plan du contrôle plurinormatif. Nous concluons en formulant l'hypothèse que tous les illégalismes sont potentiellement des «illégalismes privilégiés» (Acosta, 1988), une conclusion qui pourrait fournir des éléments dans le développement d'une éventuelle «théorie générale» du contrôle social, si tant est qu'une telle chose soit effectivement possible et souhaitable ${ }^{3}$.

\section{La criminologie avant la criminalité en col blanc: la critique de Sutherland aux théories étiologiques centrées sur la "pauvreté »}

Avant les écrits de Sutherland sur la criminalité en col blanc, la criminologie est un champ d'études discutable. La grande majorité des criminologues utilisent la prison comme laboratoire sans trop se poser de questions (Sutherland, 1931). Ils se fient aux statistiques criminelles sans considérer ce que la nouvelle criminologie des années 1970 a appelé

3. Je ne crois pas en la possibilité d'une «théorie générale» du crime, que ce soit du point de vue du comportement criminel ou de la réaction sociale. 
l'universalisation du crime et la sélectivité de la justice (Taylor, Walton et Young, 1974; Young, 1999) ${ }^{4}$. Le premier article de Sutherland (1940) sur le thème anticipe en grande partie les critiques que les criminologues des années 1970 ont formulées à l'encontre de la criminologie positiviste. «White-Collar Criminality» (Sutherland, 1940) est plus qu'un article sur les relations entre le crime et les affaires, c'est une critique de la criminologie de l'époque. La thèse principale de cet article consiste à remettre en question l'association entre la pauvreté et la criminalité en attirant l'attention sur le comportement criminel des élites:

The thesis of this paper is that the conception and explanation of crime which have just been described are misleading and incorrect, that crime is, in fact, not closely correlated with poverty or with the psychopathic and sociopathic conditions associated with poverty, and that an adequate explanation of criminal behavior must proceed along different lines. The conventional explanations are invalid principally because they are derived from biased samples. The samples are biased in that they have not included vast areas of criminal behavior of persons not in the lower class.

(Sutherland, 1940, p. 2)

Les écrits de Sutherland sur les crimes en col blanc inaugurent un nouveau domaine d'études criminologiques et contribuent au développement des sciences sociales, mais nous voulons insister sur sa «critique» de la criminologie de son époque, car cela nous permet d'interpréter ses écrits comme une application de sa théorie de l'association différentielle (Sutherland, 1947, 1983). Sutherland est d'ailleurs explicite sur cet aspect lorsqu'il pose l'hypothèse appelée à guider ses travaux jusqu'à son chef-d'œuvre White Collar Crime (1983). Selon lui, la théorie de l'association différentielle permet d'expliquer à la fois la criminalité des élites comme celle des classes inférieures ${ }^{5}$. Nous pouvons aussi facilement considérer le chapitre 15 de son livre, $A$ Theory of White

4. «The revisionism of the early 1970 s period pointed to the endemic nature of crime (universality), and emphasized the systemic class bias in the focus of the criminal justice system (selectivity). And if universality made conventional positivist notions of causation unlikely, selectivity pointed to fundamental problems in neo-classist ideas of equality before the law» (Young, 1999, p. 42).

5. «The hypothesis which is here suggested as a substitute for the conventional theories is that white-collar criminality, just as other systematic criminality, is learned; that it is learned in direct or indirect association with those who already practice the behavior; and that those who learn this criminal behavior are segregated from frequent and intimate contacts with law-abiding behavior. Whether a person becomes a criminal or not is determined largely by the comparative frequency and intimacy of his contacts with the two types of behavior. This may be called the process of differential association. It is a 
Collar Crime (p. 240-257), comme l'une des versions définitives de sa théorie de l'association différentielle pensée comme une théorie générale sur la criminalité. Afin de ne pas trahir sa pensée, mentionnons cependant que Sutherland hésite à conclure à la complétude et à l'universalité de sa théorie, mais il prétend tout de même qu'elle est «meilleure» que les autres théories générales disponibles sur le marché criminologique de l'époque ${ }^{6}$.

Si Sutherland est le premier à mettre en lumière le traitement administratif de la criminalité en col blanc, il n'arrive pas nécessairement plus loin dans la direction de la notion de gestion différentielle des illégalismes. En fait, Sutherland part de l'objet criminologique de son époque alors que la gestion différentielle est liée à une autre façon de concevoir la criminologie. Malgré son avant-gardisme, Sutherland est resté surtout dans la criminologie du passage à l'acte, comme quelqu'un plutôt intéressé à expliquer le comportement criminel et non la réaction sociale ${ }^{7}$. En analysant les relations entre crime et affaires, Sutherland a probablement produit la théorie la plus raffinée de ce paradigme, mais il a aussi ouvert la boîte de Pandore de la criminologie: les «vrais» crimes ne sont effectivement pas toujours des crimes. Cette conclusion a été exploitée plus tard à partir du prisme de la réaction sociale.

\section{La renaissance de la criminalité en col blanc dans les années 1980 : la délinquance des élites dans le contexte des théories de la réaction sociale}

Les années 1980 ont marqué le début d'une période de redécouverte de la version originale et intégrale de l'œuvre classique de Sutherland publiée finalement en 1983, mais aussi une période de renaissance des

generic explanation both of white-collar criminality and lower class criminality» (Sutherland, 1940, p. 10-11).

6. «This hypothesis is certainly not a complete or universal explanation of white collar crime or of other crime, but it perhaps fits the data of both types of crimes better than any other general hypothesis» (Sutherland, 1983, p. 240).

7. «The thesis of this book (...) is that persons of the upper socioeconomic class engage in much criminal behavior; that this criminal behavior differs from the criminal behavior of the lower socioeconomic class principally in the administrative procedures which are used in dealing with the offenders; and that variations in administrative procedures are not significant from the point of view of causation of crimes. The causes of tuberculosis were not different when it was treated by poultices and bloodletting than when treated by streptomycin» (Sutherland, 1983, p. 7; je souligne). 
études sur la déviance des élites dans un sens plus large. Le virage paradigmatique des années 1960 et 1970 a donné un nouveau souffle aux études sur la criminalité en col blanc. La «criminologie conventionnelle» perd du terrain et les notions d'étiquetage (Becker, 1963) et de criminalisation primaire et secondaire (Schur, 1971) sont mobilisées pour expliquer le traitement administratif de la criminalité en col blanc. L'émergence des perspectives critiques en criminologie a créé l'environnement propice à la rédaction et la réception de Crimes of the powerful (Pearce, 1976), Illegal But Not Criminal (Conklin, 1977), The Rich Get Richer and the Poor Get Prison (Reiman, 1979) et d'autres travaux d'influence marxiste dans l'univers universitaire.

Alors que les relations de pouvoir au centre des institutions de contrôle social intègrent l'imaginaire criminologique de l'époque, la gestion différentielle des illégalismes fait aussi son entrée, dans ses versions les plus polémistes (p. ex. Reiman, 1979) ou les plus raffinées comme celle développée par Foucault dans La société punitive (2013) et Surveiller et Punir (1975) ou Lascoumes (1996) ${ }^{8}$. Au-delà du caractère plus contestataire des ouvrages de Pearce (1976), Conklin (1977) et Reiman (1979), les années 1980 ont produit quelques travaux sur la gestion différentielle des illégalismes plus centrés sur les dynamiques de réaction sociale, proposant à tout le moins de nuancer ce qui se passait en toute «impunité pénale». Parmi ceux-ci, les travaux de Shapiro $(1980,1984,1985)$ sur les mécanismes de contrôle contre la fraude boursière se démarquent, et ce, pour deux motifs précis: d'abord, Shapiro démontre clairement que la justice criminelle est «the road not taken» (1985) dans la gestion des illégalismes financiers; ensuite, elle est l'une des premières chercheures à étendre la notion de "poursuite» afin d'inclure les «réponses juridiques de toutes sortes», et ce, tout en conservant la spécificité de chacun des systèmes normatifs mobilisés?

8. Amicelle (2014) fait une revue intéressante des travaux sur le concept d'«illégalisme» chez Michel Foucault, sur la gestion différentielle et sur la façon dont Pierre Lascoumes l'utilise dans ses travaux sur la délinquance des élites. Notre objectif n'est pas de nous éterniser sur ces sujets, mais plutôt de discuter des contributions de Susan Shapiro, Pierre Lascoumes et Fernando Acosta sur «l'organisation et le fonctionnement de la gestion différentielle des illégalismes» (Acosta, 1988, p. 11).

9. «The term prosecution is used in this context to describe the decision to take formal legal action of any kind (civil, administrative, or criminal) against securities violators. It is not used here in its more narrow and common legal usage pertaining exclusively to criminal dispositions» (Shapiro, 1984, p. 147). Le même raisonnement était déjà présent dans sa thèse de doctorat (1980, p. 179 et s.) et cela est encore plus explicite quand elle 
Sa thèse de doctorat, Detecting Illegalities (1980), publiée en version plus concise dans l'ouvrage Wayward Capitalists (1984), est un bijou en matière de nuances et de données sur les mécanismes de contrôle utilisés par la Securities and Exchange Commission (SEC: l'Autorité des marchés financiers aux États-Unis).

Les travaux d'Acosta $(1984,1985,1988)$ et Lascoumes $(1983,1985$, 1986) à la même époque vont dans le même sens que Shapiro en mettant l'accent sur la gestion différentielle et plurinormative, mais dans une perspective plus foucaldienne et théorique. En fait, Acosta et Lascoumes contribuent à notre compréhension des illégalismes liés aux domaines de l'économie et de la politique. L'excellent article d'Acosta (1988) à propos des «illégalismes privilégiés $»^{10}$ propose d'expliquer de façon globale la gestion différentielle des illégalismes, que ceux-ci soient associés aux crimes en col blanc ou non. De façon plus spécifique, il établit une distinction entre deux formes d'illégalismes: les typiques et les privilégiés. Les illégalismes typiques sont ceux qui «du moins en principe, relèvent en exclusivité d'un système normatif donné» (Acosta, 1988, p. 12), alors que les illégalismes privilégiés sont définis d'une façon beaucoup plus complexe: sur le plan juridique, ils peuvent être encadrés par différents systèmes normatifs, «notamment le droit pénal [criminel], le droit civil et, tout particulièrement, le droit administratif»; sur le plan des évènements, ce sont des situations relativement analogues à celles qui sont définies comme des crimes; et sur le plan du règlement des conflits, «ces situations conflictuelles disposent, en principe, d'un large éventail de modes de règlement (ententes à l'amiable, avertissements, sommations, sanctions disciplinaires, amendes statutaires à caractère symbolique, réparations civiles, etc.)» (Acosta, 1988, p. 13-14). Acosta laisse supposer alors une nuance importante: contrairement à la définition proposée par Foucault (2013; voir note 10), la raison pour laquelle ces illégalismes sont "privilégiés» est la «large disponibilité des modes de résolution et pas nécessairement les rapports éventuels de ce type d'illégalismes aux individus plus fortunés (Acosta, 1988, p. 14 et s.).

parle des «multiples docketing», les registres multiples des différentes procédures judiciaires, dans son chapitre méthodologique (1980, p. 95 et s.).

10. À ne pas confondre avec les «illégalismes des privilégiés» de Foucault (2013) dans le cours La société punitive, même si la notion d'Acosta fait référence à la discussion sur illégalisme et délinquance dans Surveiller et Punir (Foucault, 1975), le terme «privilégié» n'est pas présent dans ce livre. 
Les travaux de ces trois auteurs condensent, à mon avis, les grandes contributions des écrits sur la criminalité en col blanc sur le plan de la réaction sociale et surtout à propos de ce qui se passe généralement lorsque certains «crimes» ne sont pas criminalisés. Il est évident qu'il y a d'autres références remarquables dans les années 1980, par exemple le livre de Braithwaite (1984) sur l'industrie pharmaceutique, mais les travaux de Shapiro et le dialogue entre Acosta et Lascoumes amènent la discussion à un autre niveau, au-delà de la thématique de la criminalité en col blanc. Ils travaillent dans le registre de tous les types de réponses juridiques. Cela est encore plus explicite dans l'article sur les «illégalismes privilégiés» d'Acosta (1988) parce que la distinction qu'il fait entre illégalismes typiques et privilégiés ne relève pas du système normatif criminel. Il n'est pas surprenant qu'Acosta ait utilisé ce concept pour analyser la violence policière, surtout dans ses conférences pendant les années 1990, car le «privilège» est associé à la gestion plurinormative du conflit, généralement via le droit administratif. En effet, comme nous l'indiquerons bientôt, l'idée selon laquelle ces illégalismes seraient privilégiés est discutable sur deux aspects. Premièrement, la disponibilité de multiples modes de résolution des conflits n'est pas liée à l'accès à la justice ou aux choix effectués par l'accusé, mais plutôt à la mise en application des lois (enforcement) et aux choix effectués par le poursuivant. En cela, cette position se rapproche des travaux de Shapiro (1980, 1984) et de Galanter sur les dommages punitifs (1991; Galanter et Luban, 1993) ainsi que de celle de Fischer et Spire (2009) sur la gestion différentielle «comme un mode de domination assuré par des agents de l'État» (paragr. 5). Deuxièmement, la différence entre les illégalismes typiques et privilégiés ne peut pas résulter d'une distinction faite à priori par l'observateur puisqu'en principe tous les illégalismes sont passibles d'une gestion plurinormative et même ceux traditionnellement associés à la criminalité de rue.

\section{De près, les chats ne sont pas toujours gris: la criminalité typique en tant que construction plurinormative}

Si la nuit tous les chats sont gris, quand on les regarde de plus près, ils sont multicolores. Le même raisonnement s'applique à la criminalité. Les études sur les crimes en col blanc nous le donnent à penser d'une façon encore plus nuancée à partir des années 1980. L'imaginaire cri- 
minologique accepte alors relativement bien que les crimes en col blanc ne soient pas toujours des crimes quand ils sont observés de près. En effet, le débat se présente autrement puisqu'on prétend alors que même sans être techniquement considérés comme des crimes, les crimes en col blanc devraient être considérés comme de «vrais crimes», pour reprendre les mots de Sutherland (1940, 1945, 1983). Ce débat est moins important aujourd'hui parce que l'objet de la criminologie n'est plus remis en question à partir de la distinction entre crime et noncrime (Pires, 1993; Van Outrive, 1995). Depuis les années 1970, la criminologie étudie explicitement des objets qui ne sont pas toujours des crimes. Comme Shearing l'a proposé dans les années 1980 en parlant des études sur la police, il faut plutôt penser à «décriminaliser la criminologie», car le crime n'est plus la catégorie qui unifie l'objet de la discipline (Shearing, 1989). Rétrospectivement, on peut considérer que la criminologie a en effet été décriminalisée progressivement par la suite et davantage au xxI ${ }^{\mathrm{e}}$ siècle par l'ajout de nouvelles perspectives telles que la zémiologie (Bertrand, 2008; Ericson, 2007; Hillyard, Pantazis et Tombs, 2004; Vanhamme, 2010) et la justice préventive (Ashworth et Zedner 2014b; Ashworth, Zedner et Tomlin, 2013). En quelque sorte, les études sur les crimes en col blanc ont toujours été à l'avant-garde de ces enjeux disciplinaires. Notre objectif consiste maintenant à poser aussi un regard sur les autres crimes, ceux que la criminologie considère comme étant de «vrais crimes», soit la criminalité de rue. Pour ce faire, nous examinerons quelques exemples empiriques tirés de nos travaux antérieurs.

Nous avons établi les premiers liens entre les illégalismes privilégiés et la criminalité de rue dans le contexte d'une observation ethnographique de longue durée à la Commission d'immigration et du statut de réfugié (CISR), un tribunal s'autoproclamant comme étant «le plus important tribunal administratif indépendant au Canada». Au Canada, comme c'est le cas aussi dans d'autres pays, le contrôle de l'immigration relève du droit administratif et non nécessairement du droit criminel, même si certaines situations assujetties au contrôle de l'immigration peuvent être considérées comme des crimes à une autre étape du processus de réaction sociale. Les conflits liés à l'immigration font donc partie de ces illégalismes susceptibles de «bénéficier» de la gestion différentielle fondée sur le statut particulier du délinquant, soit sa condition d'étranger. Par exemple, un immigrant arrêté par la police pour avoir commis un vol peut être assujetti au processus de crimina- 
lisation, au contrôle de l'immigration, aux deux processus, ou même à aucun d'entre eux si le policier choisit de le libérer sans entreprendre d'autres démarches. Le processus de mise en forme en droit de l'immigration est très différent de celui qui prévaut dans le système de justice criminelle, la criminalisation, cette dernière ayant été fort bien décrite par Ericson (1981), Lévy $(1984,1985)$ et Acosta (1987) dans des contextes différents. La transformation de l'évènement en infraction dans le système d'immigration passe typiquement par une autre chaîne de production des illégalismes comprenant, au premier chef, l'Agence des services frontaliers du Canada (ASFC), laquelle concentre les fonctions de police, procureur, juge (dans le cas des non-résidents) et administrateur carcéral, et la CISR, qui est compétente pour les résidents permanents et les demandeurs d'asile. Il y a parfois des communications entre cette chaîne de production des illégalismes et celle de la criminalisation, mais ces processus sont «relativement autonomes» (Bourdieu, 1986, 1991; Falk-Moore, 1973, 1978) sur les plans opérationnel, juridique et institutionnel. Ainsi, la même situation-problème impliquant un étranger peut déclencher différents processus de réaction sociale qui mobilisent différents systèmes normatifs. Ceci étant dit, comme nous le verrons bientôt, les cas dont la CISR est saisie proviennent souvent d'autres systèmes normatifs, habituellement la justice criminelle, mais parfois aussi le droit pénal réglementaire (p. ex., lorsque les comportements visés ont d'abord fait l'objet d'une poursuite pour la violation d'un règlement municipal).

Contrairement aux crimes en col blanc, ces illégalismes privilégiés ne sont cependant pas sous-pénalisés, mais plutôt surpénalisés. En effet, dans certains cas, le régime d'immigration vient se superposer à la criminalisation d'une situation-problème, alors que dans d'autres, certaines situations qui n'auront pas pu se transformer en crimes en raison du caractère insuffisant de la preuve recueillie ou encore pour d'autres motifs d'ordre processuel, bénéficieront d'une mise en forme administrative, multipliant les effets punitifs (Velloso, 2013b, 2014). Dans le domaine de l'immigration, le fait que plusieurs modes de résolution des conflits sont disponibles constitue clairement un privilège pour la poursuite (l'accusation) et non pour l'accusé. En fait, le privilège de la gestion différentielle des illégalismes appartient au poursuivant qui peut choisir le ou les systèmes normatifs qui conviennent le mieux à ses intérêts. Et dans le cas du droit administratif, les avantages de cette polysémie juridique sont indéniables puisque tant la définition de la 
criminalité que le processus de mise en forme sont plus favorables au poursuivant.

D'abord, la notion de «criminalité» en droit de l'immigration est indépendante de celle de la justice criminelle en ce que c'est le soussystème juridique de l'immigration qui détermine la validité et le sens des catégories qu'il utilise et non le sous-système juridique de la justice criminelle. Suivant cette logique, la définition de criminalité est liée aux définitions du Code criminel (criminalisation primaire), mais la transformation des évènements en crime (criminalisation secondaire) n'est pas nécessaire parce que le régime d'immigration suit les règles et les principes de mise en forme du droit administratif. Par exemple, tel que nous le verrons plus loin, le système administratif impose des standards de preuve moins élevés et n'exige pas la preuve d'une faute subjective sur le plan de la responsabilité, ce qui augmente la portée de ce qui peut être traduit juridiquement comme un illégalisme.

Ainsi, nous observons que la CISR a très souvent affaire à des situations de criminalité de rue, notamment lorsqu'elle est appelée à se prononcer sur des cas de renvois pour des motifs liés à la «criminalité». Bien que nous ayons effectivement observé quelques cas allégués ou avérés de fraude, surtout des cas de fraude mineurs, tels que des faux chèques ou des vols de numéros de carte de crédit, ceux-ci forment l'exception plutôt que la règle. La plupart du temps, les illégalismes liés au volet «criminel» sont des cas de possession et de trafic de drogues, d'agression, de voies de fait, de vols ou encore le fait d'être «membres de gangs de rue». Or, la catégorie «gangs de rue» en immigration est assez fluide. La Loi sur l'immigration et la protection des réfugiés (LIPR) prévoit trois catégories de «criminalité» pour établir l'interdiction de territoire: "grande criminalité» (s.36 (1)), «criminalité» (s.36 (2)) et «activités de criminalité organisée» (s.37 (1)). Curieusement, en pratique, la catégorie «gangs de rue» est plutôt interprétée comme relevant de la «grande criminalité» et très rarement comme étant de la «criminalité organisée». Cela la distancie de la notion criminelle de "gangstérisme» qui est associée à «organisation criminelle» au sens du Code criminel, L.R.C. (1985), c. C-46, art. 467.1 (1) et 467.11 à 467.13.

Cette confusion classificatoire entre la notion de «criminalité» et celle de «gangs de rue» en immigration se produit en raison de leur apparente référence aux catégories existantes en matière criminelle et $\mathrm{du}$ fait que les règles de preuve et de responsabilité applicables en droit administratif, particulièrement en droit de l'immigration, sont très 
différentes de celles applicables en droit criminel. Selon la Loi sur l'immigration et la protection des réfugiés, la CISR «n'est pas liée par les règles légales ou techniques de présentation de la preuve» et "peut recevoir les éléments qu'elle juge crédibles ou dignes de foi en l'occurrence et fonder sur eux sa décision» (LIPR, s.175). En outre, les éléments de preuve présentés par l'accusation (l'ASFC) sont perçus comme étant plus crédibles que ceux de la défense. La crédibilité de la preuve produite par le gouvernement est rarement remise en question. $\mathrm{Si}$ l'ASFC dit une chose et l'immigrant en dit une autre, généralement la CISR décidera que la version de l'immigrant manque de crédibilité. Même si la version de l'immigrant est considérée valide, le niveau de preuve exigé est beaucoup plus bas que la norme de preuve applicable en droit criminel (hors de tout doute raisonnable), et le critère de responsabilité applicable est celui de la responsabilité stricte (un régime de faute présumée) et non la mens rea subjective. L'accusé sera ainsi présumé négligent selon la «prépondérance des probabilités» ou même selon des niveaux de preuve encore plus bas tels que "possibilité sérieuse» ou «motifs raisonnables de croire» (quelque chose d'un peu plus certain que le «simple soupçon»). Étrangement, la norme de preuve de «motifs raisonnables de croire» s'applique précisément dans les cas d'interdiction de territoire (mesures de renvoi) et de détention et mise en liberté (LIPR, ss. 33 à 43 et 54 à 61), c'est-à-dire aux situations-problèmes qui sont passibles de criminalisation et traditionnellement administrées par la justice criminelle.

Finalement, l'existence d'un acte ou d'un évènement n'est pas nécessaire pour la construction de l'infraction en immigration, car comme l'indique bien l'article 33 de la LIPR: «Les faits - actes ou omissions - mentionnés aux articles 34 à 37 sont, sauf disposition contraire, appréciés sur la base de motifs raisonnables de croire qu'ils sont survenus, surviennent ou peuvent survenir.» C'est une approche fort astucieuse de la part de l'État: les immigrants sont présumés responsables pour quelque chose qu'ils n'ont pas nécessairement encore fait et l'ASFC, qui est présumée crédible et concentre les fonctions de police et de poursuite, doit simplement faire la preuve qu'elle a des motifs raisonnables de croire que les faits peuvent se produire à un moment donné. En fin de compte, il y a des situations qui pourraient être judiciarisées par la justice criminelle et devenir un crime, mais elles finissent par prendre la voie administrative. 
Prenons l'exemple des situations-problèmes liées aux membres des gangs de rue afin d'illustrer la complexité de la réaction sociale quand on la regarde de plus près. Ces évènements, et en théorie tous les évènements, sont polysémiques sur le plan juridique et peuvent être classifiés à partir de différents systèmes normatifs et sujets à une gestion différentielle. Nous avons observé au moins six configurations de réaction sociale liées aux gangs de rue à la CISR:

A) La première correspond à une situation analogue aux illégalismes typiques décrite par Acosta (1988, voir p. 12): la réaction sociale est effectuée exclusivement par le régime d'immigration, soit parce qu'il n'y a pas assez de preuves pour justifier le dépôt d'une accusation par la police ou une poursuite criminelle, soit parce que l'ASFC s'occupe du cas depuis le début.

B) Dans la deuxième configuration, l'illégalisme prend un chemin hybride composé par la justice criminelle et l'immigration. Ainsi, la police peut déposer des accusations lors d'une enquête, mais ces accusations n'aboutissent pas à des poursuites criminelles, soit en raison de l'absence de preuve ou parce que le régime d'immigration prend le relais de la justice criminelle. Il y a bel et bien deux flux de judiciarisation en parallèle, mais le régime d'immigration avance plus vite que le régime criminel. Par contre, c'est la prise en charge par la police, le début de la mise en forme pénale (Acosta, 1987), qui a déclenché le processus en immigration par l'ASFC comme procureur.

C) La troisième configuration s'apparente à la deuxième: le cas est mené parallèlement dans les deux régimes, une poursuite criminelle est déposée, mais il n'y a pas de condamnation (grosso modo pour les mêmes raisons énumérées ci-dessus: absence de preuve ou temporalité du processus). Dans ces cas cependant, c'est le dépôt d'accusations criminelles qui déclenche la réaction en immigration. Étant donné que les normes de preuve et de responsabilité sont plus basses en droit de l'immigration (responsabilité stricte et «motifs raisonnables de croire»), les cas qui ne passent pas le test en matière pénale peuvent devenir acceptables dans le régime d'immigration (Velloso, 2013b, 2014).

D) Dans d'autres cas, il y a clairement un parcours hybride au criminel et en immigration, mais avec au moins une déclaration de culpabilité. Dans ces cas, c'est le statut de criminel ou de condamné qui déclenche la réaction sociale en immigration. 
E) Certains cas empruntent un parcours hybride, mais distinct, composé du droit pénal réglementaire et droit de l'immigration. C'est généralement le cas lorsque la personne a un dossier d'infractions réglementaires significatif. Ces cas sont relativement communs, mais difficiles à observer parce que l'utilisation de la catégorie «crime» en immigration est trompeuse. Parfois un «crime» n'est pas un crime, soit en raison des règles de preuves applicables (les configurations « $A »$ et $\langle\mathrm{B} »)$ ou bien parce que les infractions pénales réglementaires commises par les étrangers sont interprétées comme relevant de la «criminalité véritable» en matière d'immigration par les praticiens du droit à la CISR ${ }^{11}$. Ces cas sont tout de même similaires à ceux de la configuration « $\mathrm{C}$ » en ce que c'est le dossier de police qui déclenche le processus et soutient la mise en forme effectuée par l'ASFC.

F) Finalement, il y a des cas avec des trajectoires judiciaires plus complexes, formées par des allers-retours entre le droit criminel, le droit pénal réglementaire et le droit de l'immigration. Dans ces cas, les parcours de réaction sociale communiquent entre eux alors que les illégalismes se multiplient dans deux ou plusieurs systèmes normatifs. Plusieurs scénarios sont possibles, la réaction du régime d'immigration étant déclenchée par un ensemble de crimes et d'infractions réglementaires. Le cas habituel est celui d'un immigrant qui commet une infraction réglementaire en étant sous le coup d'une ordonnance conditionnelle de mise en liberté ou de probation résultant d'une accusation criminelle antérieure. Cette infraction réglementaire représente alors un non-respect des conditions et entraîne le dépôt d'accusations pour une nouvelle infraction criminelle et est ensuite soumise à une procédure de renvoi à la CISR. C'est le non-respect qui devient un crime, mais c'est l'ensemble des «crimes» qui actionne la réaction sociale en immigration. Ces cas complexes sont nombreux lorsque les procédures judiciaires s'étirent sur une longue période de temps et que les cas se retrouvent en procédure de révision du sursis de la mesure de renvoi ${ }^{12}$ à la Section d'appel de la CISR.

11. Cela est clairement une stratégie d'accusation dans les cas d'inadmissibilité (le renvoi du pays) «pour motifs de criminalité», étant donné que les agents de l'ASFC construisent un récit sur l'immigrant comme étant un «criminel» et quelqu'un qui ne s'est pas bien intégré au Canada.

12. La révision du sursis de la mesure de renvoi est l'une des dernières étapes possibles à la CISR. Le processus de renvoi commence par une enquête en matière d'immigration à 
Partant d'une situation-problème, comme l'appartenance à un gang de rue, pouvant potentiellement n'être que criminalisée dans la perspective du modèle standard en criminologie, nous retenons deux aspects de notre observation des différentes configurations de réaction sociale dans le régime d'immigration.

Premièrement, il nous a été possible d'observer la gestion différentielle des illégalismes à partir d'un régime autre que le système de justice criminelle. Nous observons la gestion des illégalismes d'immigration à partir du tribunal d'immigration, mais ce faisant, nous avons observé ce qui se passait dans les autres régimes. C'est une approche différente de celle adoptée par Shapiro $(1980,1984)$ ou Acosta (1984, 1988) qui ont fait leurs observations, soit à partir de la poursuite agissant de façon plurinormative (la SEC, dans le cas de Shapiro), soit à partir de la justice criminelle au Canada, plus particulièrement la police dans le cas d'Acosta. Étant donné que le point de départ de notre observation était la CISR, nous n'avons certainement pas pu observer toute la criminalité de rue ou encore les infractions qui n'atteignaient pas le tribunal, mais nous avons été en mesure de voir des configurations de réaction sociale plus complexes telle celle décrite dans le scénario $« \mathrm{~F} »$. Les cas de révision de sursis d'une mesure de renvoi sont de bons exemples de gestion différentielle de la criminalité de rue liée aux contrevenants étrangers.

Deuxièmement, si nous inversons le point de vue du scénario «F» et que nous observons hypothétiquement ces cas à partir de la justice criminelle, nous constatons que ce qui détermine le caractère "privilégié» d'un illégalisme est fondamentalement le contexte de la réaction sociale et la temporalité des modes de résolution des conflits utilisés et pas nécessairement le type de criminalité (p. ex. les crimes en col blanc). La criminalité de rue ne peut pas être réduite à la catégorie de crimes, des illégalismes typiques qui «relèvent en exclusivité d'un système normatif» (Acosta, 1988, p. 12) [1e droit criminel]. Elle est aussi composée par des infractions réglementaires, des illégalismes en matière d'immigration et peut-être d'autres qui ont échappé à notre étude. La

\footnotetext{
la Section de l'immigration où un commissaire décide si l'immigrant est interdit de territoire et donc sujet à une mesure de renvoi. Dans certains cas, l'immigrant a un droit d'appel à la Section d'appel de l'immigration où un autre commissaire peut accepter l'appel, le refuser ou bien accorder un sursis de la mesure de renvoi (c.-à-d. suspendre temporairement le renvoi, et ce, jusqu'à cinq ans), avec suivi et révision des conditions imposées dans le sursis.
} 
distinction, et l'opposition, que Foucault (1975) laisse supposer entre les illégalismes «de biens» et «de droit» ne s'applique pas; au contraire, c'est une mauvaise distinction pour penser l'économie politique des illégalismes et des sanctions dans un sens plus large. En théorie (et en pratique), le plan des évènements est toujours ouvert en termes de signifié (sens), il peut être perçu comme illégal ou pas, et un même illégalisme peut être le résultat de différentes configurations juridiques de contrôle.

\section{Le jour, tous les illégalismes sont "privilégiés": contributions à une possible «théorie générale » du contrôle social}

L'une des plus grandes contributions des études sur la délinquance des élites à la criminologie tient donc à leur capacité de nuancer la gestion des illégalismes dans différents systèmes normatifs. Sans les écrits de Sutherland, Shapiro, Acosta, Lascoumes et d'autres, nous ne pourrions probablement pas percevoir la gestion plurinormative de la criminalité de rue à partir du régime d'immigration. Comme Clifford Geertz nous l'a enseigné: «l'interprétation n'est pas la seule à côtoyer l'observation la plus immédiate: la théorie, dont une telle interprétation dépend sur le plan conceptuel, est dans la même situation». Nous pourrions continuer en le paraphrasant: "le modèle de la "confusion des langues [juridiques]" n'est pas une idée qui nous est venue avec [les cas observés à la CISR]. C'est une idée que des collègues, des étudiants et nos prédécesseurs nous ont apprise et que nous avons introduite dans cette étude» (Geertz, 2003, p. 230). Les crimes en col blanc et les illégalismes privilégiés nous ont donc permis d'observer et de penser la criminalité de rue, le système pénal et la criminologie autrement.

Si dans les années 1960 et 1970 la nouvelle criminologie a donné à penser que les crimes n'existaient pas en soi et qu'ils étaient construits par le système pénal, aujourd'hui, avec la radicalisation de la notion d'illégalisme proposée ci-dessus, on peut laisser supposer que le système pénal se construit en même temps qu'il construit la délinquance. Le pénal, entendu dans son sens large comme étant le domaine de la gestion des illégalismes avec une possibilité d'imposition de «châtiments légaux» (Foucault, 1975), n'existe pas en soi d'une manière plus ou moins fixe. Il se forme lors de la réaction sociale, en prenant différentes configurations structurelles et en produisant différents types 
d'illégalismes, y compris, mais non exclusivement, des crimes. Les crimes peuvent être interprétés comme des formes très particulières d'illégalismes et sans aucune garantie d'être un illégalisme typique dans le sens où l'entend Acosta (1988), soit le produit exclusif d'un seul système normatif (criminel). C'est seulement après l'ensemble de la réaction sociale qu'il est possible d'établir si un crime est en fait exclusivement un crime ou une construction plurinormative. On peut essayer de prédire certaines configurations pénales, mais cela est très difficile, car le pénal s'adapte comme un mobile, comme une structure dynamique (Velloso, 2013a, 2014). Nous avons proposé plus tôt six configurations de réaction sociale, mais il y a évidemment plusieurs autres possibilités si l'on ajoute les régimes civils (p. ex. une ordonnance préventive de ne pas s'approcher d'un conjoint) ou si l'on considère les commissions de libération conditionnelle en tant que systèmes normatifs distincts et relativement autonomes - en fait, au Canada, ces commissions sont des tribunaux administratifs et donc relativement autonomes, même sur le plan institutionnel.

Dans les années 1940, Sutherland a utilisé l'exemple de la criminalité en col blanc pour promouvoir sa théorie de l'association différentielle comme étant la meilleure théorie générale sur les crimes. La réaction sociale n'était pas l'objet de la criminologie de son époque, mais rétrospectivement ses études ont préparé le terrain pour l'idée de gestion différentielle et les nuances développées à partir des années 1980. Le modèle des illégalismes privilégiés proposé par Acosta (1988) est plus qu'un outil analytique pour interpréter les illégalismes du monde des affaires, de l'administration publique, de la santé publique, de l'environnement et de la sécurité au travail. La proposition d'Acosta est une espèce de théorie générale sur le contrôle social. Son modèle aurait pu prétendre expliquer la réaction sociale à tous les illégalismes, mais il opérait encore à partir de la distinction foucaldienne entre «illégalismes de biens» et «illégalismes de droit» ou plutôt entre «atteintes patrimoniales» et «atteintes à la vie» (Acosta, 1988, p. 15-16). Il faut cependant noter qu'Acosta a tenté de garder son modèle le plus ouvert possible et indépendant de la catégorie de crime, une grande avancée pour la criminologie de l'époque. Ce faisant, ses travaux auront été les précurseurs de certaines notions présentes dans les écrits de Shearing sur la gouvernance nodale (Burris, Kempa et Shearing, 2008; Shearing, 1996; Shearing et Johnston, 2010; Shearing et Wood, 2003, 2007). Or, son modèle présentait deux limites sur le plan théorique. 
D’abord, celui-ci avait été pensé en fonction des crimes en col blanc et faisait des distinctions approximatives entre trois systèmes normatifs (criminel, civil et administratif). Ensuite, le modèle avait été pensé d'une façon relativement fixe et était décrit à partir des interactions et des superpositions entre les trois systèmes représentés sous forme de cercles, telle la théorie des ensembles (Figure 1).

\section{FIG URE 1}

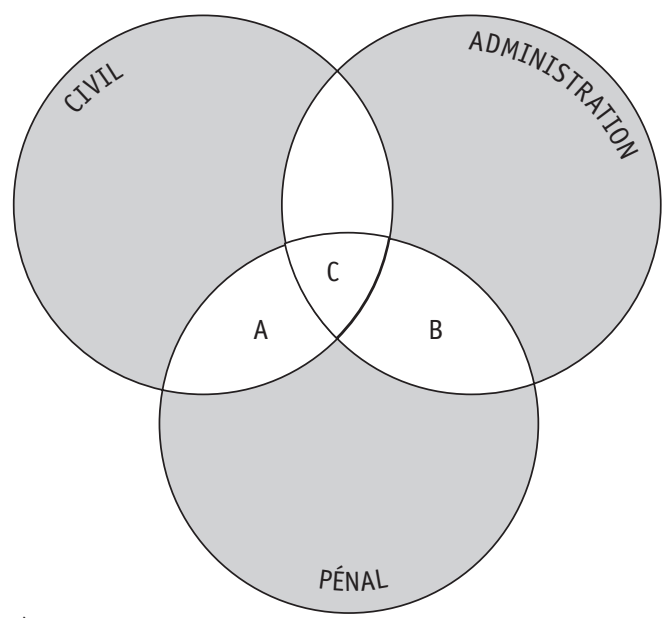

(Acosta, 1988, p. 12)

La première limite nous semble problématique parce que les distinctions entre les systèmes normatifs relevés ne sont pas assez précises pour nuancer la gestion des illégalismes. Par exemple, où se situe le droit pénal réglementaire dans ce modèle, et comment pouvons-nous distinguer opérationnellement les régimes distincts et relativement autonomes qui forment l'ensemble «administratif» et qui peuvent participer à la réaction sociale punitive, notamment le droit de l'immigration, le droit militaire, les commissions de libérations conditionnelles, la propriété intellectuelle, etc. ? Il faut noter que le texte de 1988 d'Acosta offre la première et la seule version de son modèle d'analyse. Il est fort possible qu'Acosta fût arrivé à faire de telles distinctions s'il avait continué à développer son modèle. La deuxième limite est cependant plus importante, car la représentation du modèle implique que ces systèmes normatifs existent en soi. Acosta adopte l'approche constructiviste de l'époque selon laquelle certains crimes existent parce qu'il y a un sys- 
tème pénal, mais il le fait d'une façon encore plus sophistiquée: selon lui, il y a des illégalismes privilégiés parce qu'il y a trois systèmes normatifs. Or, comme nous l'avons indiqué dans la section précédente, les interactions entre ces systèmes normatifs (trois ou plus) créent des configurations variées et variables tout au long de la réaction sociale. Il faut plutôt penser à un modèle dynamique pour rendre compte de ces variations parce que c'est le pénal lui-même qui se construit et se reconstruit constamment. Par conséquent, il nous semble plus approprié sur le plan descriptif de représenter ce modèle comme un mobile (Figure 2) et non comme des cercles référant à la théorie des ensembles (Figure 1).

\section{FIG URE 2}

\section{Le pénal comme un mobile}

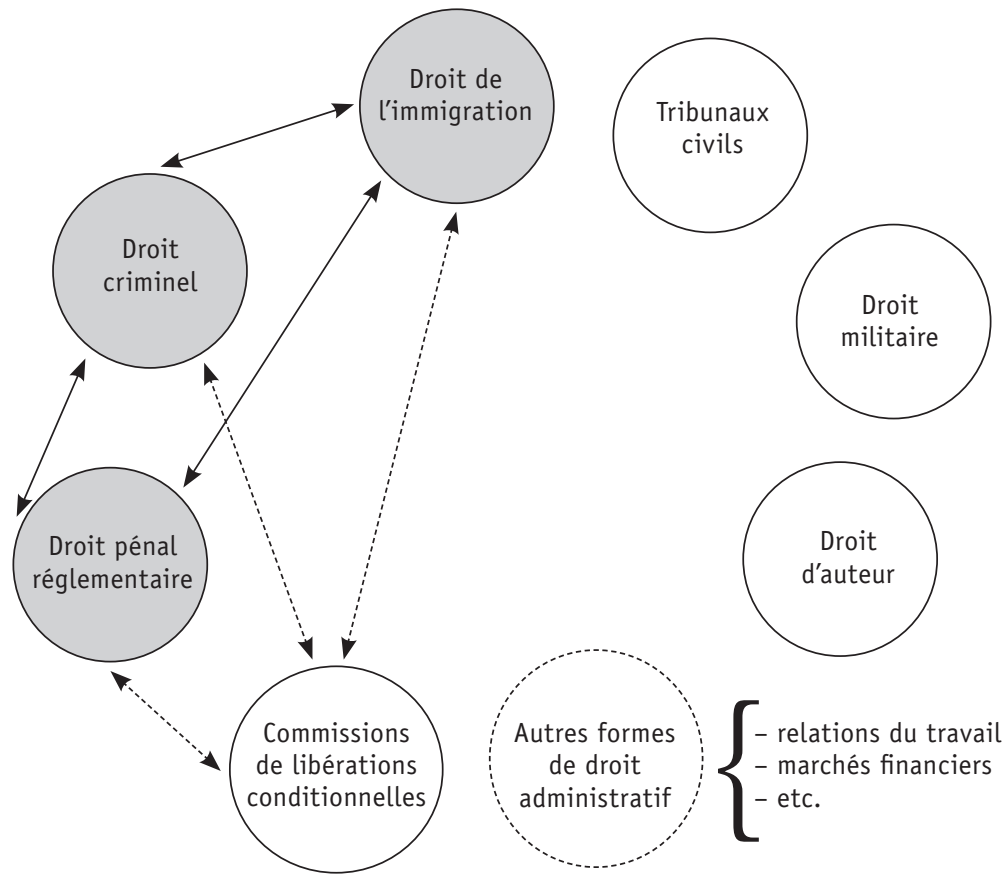

(Adapté de Velloso, 2014, p. 78)

Penser le pénal comme un mobile est un développement plus ouvert du modèle de gestion des illégalismes privilégiés proposé par Acosta. Ce modèle pourrait être mobilisé pour analyser le contrôle social (et la 
gestion différentielle) de n'importe quel illégalisme. Un mobile, par définition, n'est centré sur aucune de ses parties puisqu'il est constamment en mouvement. La particularité du pénal perçu comme un mobile est que le pénal n'existe pas, il se construit selon le contexte et c'est alors un modèle qui laisserait plutôt supposer la coexistence de plusieurs théories particulières du contrôle social à différentes échelles et juridictions (Valverde, 2009, 2015).

Au-delà de sa dimension dynamique et ouverte, il faut noter qu'il y a dans ce second modèle plus de systèmes normatifs représentés que dans le modèle des illégalismes privilégiés (Acosta, 1988). Cela ne veut pas dire que le modèle d'Acosta est incomplet, mais plutôt que nous réalisons d'autres distinctions entre les systèmes normatifs afin de mieux nuancer les processus de réaction sociale et de gestion différentielle des illégalismes. Par exemple, au lieu d'utiliser le droit administratif comme une unité, nous utilisons au moins cinq unités fondées sur le droit administratif canadien: droit de l'immigration, commissions de libération conditionnelle, droit militaire, droit d'auteur et propriété intellectuelle, et une unité ouverte pour couvrir les «autres formes de droit administratif», telles celles liées aux relations de travail, aux marchés financiers, etc. Nous considérons tous ces sous-systèmes juridiques comme des systèmes normatifs distincts parce qu'ils sont relativement autonomes sur le plan opérationnel, même s'ils sont encadrés par des règles générales en droit administratif et par certaines décisions des tribunaux supérieurs ${ }^{13}$. Bref, le droit administratif pratiqué à la Commission d'immigration et du statut de réfugié est très différent du droit pratiqué à la Commission québécoise des libérations conditionnelles, à l'Autorité des marchés financiers ou à la Commission des relations du travail.

Nous n'utilisons pas l'unité «pénal» d'Acosta pour des raisons similaires. Finalement, notons que nous avons placé des flèches continues entre les systèmes normatifs droit criminel, droit pénal réglementaire et droit de l'immigration pour représenter les flux de réaction sociale

13. Par exemple, la norme de contrôle judiciaire en droit administratif canadien en vigueur depuis 1999 a changé récemment avec l'arrêt Dunsmuir (Dunsmuir c. NouveauBrunswick, [2008] CSC 9). Dunsmuir est à l'origine un cas de relations de travail, mais cette décision de la Cour suprême a changé, en théorie, les normes de contrôle judiciaire pour toutes les formes de droit administratif. Un an plus tard, la Cour a précisé les critères établis en Dunsmuir dans l'arrêt Khosa (Canada [Citoyenneté et immigration] c. Khosa [2009] SCC 12), un cas de droit d'immigration et ces précisions sont également valides, en théorie, dans l'ensemble des sous-systèmes administratifs. 
documentés dans notre étude (les configurations hybrides de «B» à $« \mathrm{~F} »)$. Les flux entre les commissions de libération conditionnelle et les autres systèmes normatifs échappent à la portée de notre étude, mais nous avons décidé de les représenter avec des flèches pointillées parce que ces parcours de réaction sociale sont logiques et même indirectement observables dans quelques cas des configurations «B» et « $\mathrm{F}$ ». Nos données laissent supposer qu'ils existent en pratique, mais une recherche plus approfondie serait nécessaire pour mieux décrire ces flux internormatifs. Enfin, la littérature scientifique discutée dans la partie 3 de l'article nous donne à penser qu'il est possible d'établir d'autres liens entre les différents systèmes normatifs décrits dans le modèle du pénal comme un mobile ${ }^{14}$, mais nous avons décidé de mettre en évidence seulement les flux et les trois systèmes normatifs (en gris) abordés dans notre étude.

Ce que nous avons tenté de conceptualiser, c'est une théorie du contrôle social qui valorise comment différents systèmes normatifs interagissent lors de la gestion des illégalismes et de la construction de la déviance, dont la criminalité de rue. Notre contribution se résume ici à souligner l'apport considérable des études sur les crimes en col blanc pour la criminologie dans ce sens. Celles-ci continuent à problématiser la criminologie et à élargir son «horizon» de possibilités (Luhmann, 1995), notamment à partir du prisme de la réaction sociale plurinormative, produisant ainsi de nouveaux sens ${ }^{15}$. Si les criminologues du xxie siècle souhaitent encore étudier la construction sociale des «vrais» crimes et criminels de près, les études sur la criminalité en col blanc offrent certainement les outils pour nuancer et raffiner substantiellement le débat.

14. Par exemple, les travaux d'Acosta et de Shapiro auxquels nous nous référons au long de l'article abordent des configurations de réaction sociale composées par le droit criminel, le civil et par les autres formes de droit administratif (marchés financiers et relations du travail). Les travaux de Galanter sur les dommages punitifs et ceux d'Ashworth et Zedner (2010, 2012, 2014a) sur les ordonnances de prévention discutent plutôt de configurations entre les régimes criminel et civil.

15. "What varies at any moment is not simply the "object" of an intention. Instead, meaning processing constantly shapes anew the meaning-constitutive difference between actuality and potentiality. Meaning is the continual actualization of potentialities. But because meaning can be meaning only as the difference between what is actual at any moment and a horizon of possibilities, every actualization always also leads to a visualization of the potentialities that could be connected up with it» (Luhmann, 1995, p. 65). 


\section{Références}

Acosta, F. (1984). Les affaires de Ville d'Anjou ou la mise en forme pénale de la corruption politique (Thèse de doctorat inédite). Université de Montréal, Canada.

Acosta, F. (1985). La corruption politico-administrative: émergence, constitution et éclatement d'un champ d'études. Déviance et société, 9(4), 333-354.

Acosta, F. (1987). De l'évènement à l'infraction: le processus de mise en forme pénale. Déviance et société, 11(1), 1-40.

Acosta, F. (1988). À propos des illégalismes privilégiés. Réflexions conceptuelles et mise en contexte. Criminologie, 21(1), 7-34.

Amicelle, A. (2014). 'Deux attitudes face au monde': La criminologie à l'épreuve des illégalismes financiers. Cultures er Conflits, 94-95-96, 65-98.

Ashworth, A. et Zedner, L. (2010). Preventive Orders: A Problem of UnderCriminalization? Dans R. A. Duff, L. Farmer, S. Marshall, M. Renzo et V. Tandros (dir.), The Boundaries of the Criminal Law (p. 59-87). Oxford, Royaume-Uni: Oxford University Press.

Ashworth, A. et Zedner, L. (2012). Prevention and Criminalization: Justifications and Limits. New Criminal Law Review, 15(4), 542-571.

Ashworth, A. et Zedner, L. (2014a). Civil Preventive Orders. Dans A. Ashworth et L. Zedner (dir.), Preventive Justice (p. 74-94). Oxford, Royaume-Uni : Oxford University Press.

Ashworth, A. et Zedner, L. (dir.) (2014b). Preventive Justice. Oxford, RoyaumeUni: Oxford University Press.

Ashworth, A., Zedner, L. et Tomlin, P. (dir.) (2013). Prevention and the Limits of the Criminal Law. Oxford, Royaume-Uni: Oxford University Press.

Becker, H. S. (1963). Outsiders: Studies in the Sociology of Deviance. New York, NY: Free Press.

Bertrand, M. A. (2008). Nouveaux courants en criminologie: "études sur la justice" et "zémiologie". Criminologie, 41(1),177-200.

Bourdieu, P. (1986). La force du droit: Éléments pour une sociologie du champ juridique. Actes de recherche en sciences sociales, 64, 3-19.

Bourdieu, P. (1991). Les juristes, gardiens de 1'hypocrisie collective. Dans F. Chazel et J. Commaille (dir.), Normes juridiques et régulation sociale (p. 95-99). Paris: LDGJ.

Braithwaite, J. (1984). Corporate Crime in the pharmaceutical Industry. Londres, Royaume-Uni: Routledge.

Burris, S., Kempa, M. et Shearing, C. (2008). Changes in Governance: A Cross-Disciplinary Review of Current Scholarship. Akron Law Review, 4(1), 1-66.

Conklin, J. E. (1977). Illegal But Not Criminal: Business Crime in America. Englewood Cliffs, NJ: Prentice Hall.

Debuyst, C., Digneffe, F. et Pires, A. (dir.) (1998). Histoire des savoirs sur le crime et la peine 2: la rationalité pénale et la naissance de la criminologie. Bruxelles, Belgique: Larcier. 
Ericson, R. (1981). Making Crime. Toronto. Ontario: Butterworth.

Ericson, R. (2007). Crime in an Insecure World. Cambridge, Royaume-Uni : Polity Press.

Falk-Moore, S. (1973). Law and Social Change: The Semi-Autonomous Social Field as an Appropriate Subject of Study. Law E Society Review, 7(4), 719746.

Falk-Moore, S. (1978). Law as Process: An Anthropological Approach. Londres, Royaume-Uni : Routledge.

Foucault, M. (1975). Surveiller et punir: Naissance de la prison. Paris, France: Gallimard.

Foucault, M. (2013). La société punitive: Cours au Collège de France, 1972-1973. Paris, France: Seuil/Gallimard.

Galanter, M. (1991). Punishment, Civil Style: Punishment Outside the Criminal Law in the Contemporary United States. Israel Law Review, 25, 759-78.

Galanter, M. et Luban, D. (1993). Poetic Justice: Punitive Damages and Legal Pluralism. The American University Law Review, 42, 1393-1463.

Geertz, C. (2003). La description dense - vers une théorie interprétative de la culture. Dans D. Céfaï (dir.), L'enquête de terrain. Paris, France: La découverte, 208-233.

Hillyard, P., Pantazis, C. et Tombs, S. (dir.) (2004). Beyond Criminology. Londres, Royaume-Uni: Pluto.

Hulsman, L. (1986). Critical criminology and the concept of crime. Contemporary Crises, $10(1), 63-80$.

Hulsman, L. et De Celis, J. B. (1982). Peines perdues: Le Système pénal en question. Paris, France: Le Centurion.

Lascoumes, P. (1983). Sanction des fautes ou gestion des illégalismes: l'hétérogénéisation du droit pénal. Un exemple de répression de la fraude fiscale. Revue interdisciplinaire d'études juridiques, 10, 125-156.

Lascoumes, P. (1985). La place du pénal dans le règlement différentiel des conflits. L’Année sociologique, 35, 153-165.

Lascoumes, P. (1986). Les affaires ou l'art de l'ombre. Paris, France: Centurion.

Lascoumes, P. (1996). L’illégalisme, outil d'analyse. Sociétés er Représentations, 3, 78-84.

Lévy, R. (1984). Pratiques policières et processus pénal: le flagrant délit. Paris, France: CESDIP.

Lévy, R. (1985). Scripta manent: la rédaction des procès-verbaux de police. Sociologie du travail, 2, 408-423.

Luhmann, N. (1995). Social Systems. Stanford, CA: Stanford University Press.

Pearce, F. (1976). Crimes of the powerful: Marxism, crime, and deviance. Londres, Royaume-Uni : Pluto Press.

Pires, A. P. (1993). La criminologie et ses objets paradoxaux: Réflexions épistémologiques sur un nouveau paradigme. Déviance et Société, 17, 129-161.

Reiman, J. H. (1979). The rich get richer and the poor get prison. Toronto, Ontario: Wiley. 
Schur, E. (1971). Labeling deviant behavior: its sociological implications. New York, NY: Harper \& Row.

Shapiro, S. P. (1980). Detecting Illegalities: A Perspective on the Control of Security Violations. (Thèse de doctorat inédite). Yale University, New Haven, CT.

Shapiro, S. P. (1984). Wayward Capitalists: Target of the Security and Exchange Commission. New Haven, CT: Yale University Press.

Shapiro, S. P. (1985). The Road not Taken: The Elusive Path to Criminal Prosecution for White-Collar Offenders. Law and Society Review, 19(2),179218.

Shearing, C. (1989). Decriminalizing Criminology: Reflections on the Literal and Tropological Meaning of the Term. Canadian Journal of Criminology, 31, 169-178.

Shearing, C. (1996). Reinventing Policing: Policing as Governance. Dans O. Manerin (dir.) Policing Change: Changing Police (p. 285-307). New York, NY: Garland Press.

Shearing, C. et Johnston, L. (2010). Nodal Wars and Network Fallacies: A Genealogical Analysis of Global Insecurities. Theoretical Criminology, 14(4), $1-20$.

Shearing, C. et Wood, J. (2003). Nodal Governance, Democracy and the New Denizens: Challenging the Westphalian Ideal. Journal of Law and Society, 30(6), 400-19.

Shearing, C. et Wood, J. (2007). Imagining security. Londres, Royaume-Uni: Willan.

Slingeneyer, T. (2005). La pensée abolitionniste hulsmanienne. Archives de politique criminelle, 27, 5-36.

Sutherland, E. H. (1931). The Prison as a Criminological Laboratory. Annals of the American Academy of Political and Social Science, 157, 131-136.

Sutherland, E. H. (1940). White-Collar Criminality. American Sociological Review, 5(1), 1-12.

Sutherland, E. H. (1941). Crime and Business. Annals of the American Academy of Political and Social Sciences, 217, 112-118.

Sutherland, E. H. (1945). Is 'White-Collar Crime' Crime? American Sociological Review, 10(2), 132-39.

Sutherland, E. H. (1947). Principles of Criminology. Chicago, IL: Chicago University Press.

Sutherland, E. H. (1983). White-Collar Crime: the Uncut Version. New Haven, CT: Yale University Press.

Taylor, I., Walton, P. et Young, J. (1974). The new criminology. New York, NY: Harper and Row.

Valverde, M. (2009). Jurisdiction and Scale: Legal “Technicalities" as Resources for Theory, Social and Legal Studies, 18(2): 139-157.

Valverde, M. (2015). Chronotopes of Law: Jurisdiction, Scale and Governance. New York, NY: Routledge. 
Van Outrive, L. (1995). La criminologie et ses objets paradoxaux: la nouveauté doit se trouver ailleurs. Déviance $\mathfrak{F}$ Société, 19(3), 279-289.

Vanhamme, F. (2010). La zémiologie: nouvelle discipline, extension du champ criminologique? Revue de droit pénal et de criminologie, Décembre, 1311-1326

Velloso, J. (2013a). Beyond criminocentric dogmatism: Mapping institutional forms of punishment in contemporary societies. Punishment $\mathcal{W}$ Society, 15(2), 166-186.

Velloso, J. (2013b). Au-delà de la criminalisation: l'immigration et les enjeux pour la criminologie. Criminologie, 46(1), 55-82.

Velloso, J. (2014). Seeking Alternatives for Criminology: the Immigration and Refugee Board Practices on the Regulation of Immigration in Canada. (Thèse de doctorat inédite). Université d'Ottawa, Canada.

Young, J. (1999). The Exclusive Society. Londres, Royaume-Uni: Sage.

\title{
The plurinormative control of street gangs: contributions of elite deviance studies to the understanding of the judicialization of street crimes
}

\begin{abstract}
Edwin Sutherland was one of the first scholars to question the association between poverty and crime that dominated criminology in the early twentieth century. He suggested not only that criminology should study white-collar crimes but also that his theory of differential association was a general theory capable of explaining all types of criminal behaviour. In this article, we make a similar argument from a social reaction perspective. We draw from elite deviance studies to explain the social control of the most visible forms of crime, especially street crimes, and suggest that the concept of "privileged illegalities" (Acosta, 1988) is useful in analyzing how penal institutions react to all kinds of illegalities. Ultimately we argue that street crimes, similar to white-collar crimes, are governed though different normative systems (e.g. administrative law, civil law, etc.). This often results in the imposition of greater punishments, especially if the offender is foreign.
\end{abstract}

KEYWORDS - Privileged illegalities, elite deviance, street gang members, immigration control, social reaction.

\section{El control plurinormativo de las pandillas callejeras. Contribuciones de los estudios sobre la delincuencia de las elites a la comprehensión de la judiciarización de la criminalidad callejera}

RESUMEN - Edwin Sutherland fue uno de los primeros en cuestionar la asociación entre pobreza y criminalidad que dominaba el campo criminológico a principios del siglo XX. Propuso no sólo que la criminología estudie los white-collar crimes, sino que además esos estudios reforzaron su teoría de la asociación diferencial como una teoría general, aplicable a todos los tipos de comportamientos criminales. En este artículo, persegui- 
mos un objetivo similar, pero sobre el control social: proponemos una reflexión sobre las formas más visibles de delincuencia, y más precisamente sobre la criminalidad callejera, partiendo de ciertos estudios sobre la reacción social a la delincuencia de las elites. Sugerimos que la noción de "ilegalidades privilegiadas" (Acosta, 1988) es apropiada para analizar todas las ilegalidades. De hecho, como los white-collar crimes, los delitos callejeros también son frecuentemente administrados por las instituciones jurídicas de manera plurinormativa, es decir apoyándose en diferentes sistemas normativos más allá y además de la justicia criminal (por ejemplo, el derecho administrativo, el derecho civil, etc.) En los hechos, esta técnica resulta a menudo en castigar más a los delincuentes señalados, sobretodo a los extranjeros.

PALABRAS CLAVE - ilegalidades privilegiadas, delincuencia de elites, miembros de pandillas callejeras, control de la inmigración, reacción social. 